\title{
Innovative launching schemes in ECR ion sources
}

\author{
Giuseppe Torrisi ${ }^{1, *}$, David Mascali ${ }^{1}$, Gino Sorbello ${ }^{1,2}$, Giuseppe Castro $^{1}$, Eugenia Naselli ${ }^{1,3}$, Ornella Leonardi ${ }^{1}$, Luigi \\ Celona $^{1}$ and Santo Gammino ${ }^{1}$
}

${ }^{1}$ INFN-Laboratori Nazionali del Sud, via S. Sofia 62, Catania, 95123, Italy

${ }^{2}$ Università degli studi di Catania, Viale Andrea Doria 6, Catania, 95125, Italy

${ }^{3}$ Dipartimento di Fisica e Astronomia, Università of Catania,Via S. Sofia, 64 I-95123 Catania

\begin{abstract}
An optimised RF power delivery in magnetoplasmas of ECR ion sources is crucial in order to provide a cost effective upgrade (plasma density and temperature increase and multiply charged ions production) of these machines without recurring to higher and higher magnetic fields, power level and pumping wave frequency. This can be done by following two strategies: a) in a pure ECR-heating scenario, by multiplexing different frequencies; b) in a modal-conversion scenario, by multiple-launching at different frequencies, controllable angles and polarization. The paper will show two typical cases in both the aforementioned scenarios, as developed at INFN-LNS. Test-benches have been developed on purpose, such as the "Plasma Reactor" and "Flexible Plasma Trap", and solutions have been proposed also for ion beams current boosting in the injectors of the Superconducting Cyclotron.
\end{abstract}

\section{Introduction}

There is great interest in the ECRIS community in increasing the efficiency of ECR heating as a way to maximize the current of extracted ions. Experiments have identified a number of useful techniques for maximizing the extracted currents, such as increasing the frequency scaling and exploiting the multiple frequency heating with the "frequency tuning" [1-4].

Proper coupling of the RF power to the plasma in an electron-cyclotron resonance (ECR) ion source is probably the most important factor in achieving satisfactory performances. Poor coupler design in fact can lead to a variety of undesirable effects such as high reflected power, low plasma density, unstable operation, and, eventually, poor performance.

Several efforts have been paid to RF coupling to ECRIS plasmas at INFN-LNS in the last decade. Most of them were devoted to Frequency Tuning Effect $[5,6]$, which is now routinely adopted for beam optimisation (both in terms of currents and phase-space parameters) in many ECRIS worldwide. Before the use of frequency tuning technique, the two/multiple frequency method proved to be a reliable way to increase the produced ion beam currents in ECRIS. The positive effect of this technique was observed for the first time already in '90s [3]. The impact was observed to be higher on the ions at higher charge states, and this was a proof that the effect produced a more efficient heating of the ionizing electrons.

Output currents and charge states are in fact related to plasma density and lifetime by the well known relations: $<q>\propto n_{e} \tau_{i}$, provided that $T_{e}$ is high enough to allow deep ionisation of atoms, and $\mathrm{I} \propto \mathrm{n}_{\mathrm{e}} / \tau_{\mathrm{i}}$, where $\mathrm{I}$ is the output current and $<q>$ the average charge state. More recently, some authors [7] have shown the use of two frequencies instead than one, even when they carry the same (but shared) amount of RF power, contributes to damping of instabilities.

The above mentioned methods can be further optimized, and some recent results relying to a very compact setup for summing up Frequency Tuning Effect (FTE) to Two Frequency Heating (TFH) in small-size ECRIS will be briefly described in the following.

Another challenging research in the ECRIS field concerns the intrinsic currents limitations affecting these machines. These rely with the well-known "omegasquared" law (due to the electromagnetic cutoff and RF scattering into the [8] cone). The only way for any performance boost, in the recent past, has been the increase of the pumping wave frequency, which in turn implies a scaling of the confining magnetic field as well. One possibility to overcome this problem is to implement in ECRIS also some modal-conversion scheme, such as XB or OXB conversion. Some attempts have been already done [9].

The optimization of the OXB conversion helps to maximize the amount of the absorbed RF power.

It is well known in the field of fusion reactors that modal-conversion require high control of RF launching into the plasma [10]: not only the power, but launching angle and wave polarisation are critical too.

The latter point is deemed as crucial for any future developments of ECRIS. Up to now, in fact, RF launching in these machines has been based on the very simple scheme of rectangular (or, more rarely, circular) waveguides mechanically matched to cylindrical plasma

Corresponding author: giuseppe.torrisi@lns.infn.it 
chambers. This scheme, despite its simplicity, cannot allow any mastering of the RF coupling with the plasma. R\&D on RF launchers for modal conversions is therefore deemed to become more and more important for any further boost of their performances.

\section{Multiplexing Two Different Frequencies in an ECR-H Scenario}

The "Two Frequency Heating" (TFH) has been implemented many times in ECRIS equipped by a double microwave-port at the injection endplate. We tried to implement the same method in a compact ECRIS named CAESAR, and operating since 2002 at INFNLNS as injector of the Superconducting Cyclotron. Due to its compactness, the plasma chamber has only one microwave port allowing microwave launching. Therefore TFH requires a proper setup to be correctly implemented.

The TFH experiment involved the use of a "diplexer Hybrid coupler" Waveguide Combiner in the frequency band [13.5-15.6] GHz, up to $1.5 \mathrm{~kW}$, (see Fig. 1).

This 4-ports passive device implements a "multiplexing" in the frequency domain, i.e. it allows to combine the power coming out from the two $500 \mathrm{~W}$ TWTs amplifiers at two different frequencies, combined on an output port which goes towards the plasma chamber.

The use of two TWT allows the contemporaneous tuning of both the frequencies, in separate phases. Once optimized both, in terms of relative maximization of the source's performances, they can be summed up by the diplexer, and launched at the same time into the plasma chamber. The remaining fourth port of the device, called "insulated port", is terminated on an adapted high-power waveguide load (up to $1 \mathrm{~kW}$ of Power Handling) in order to minimize and absorb unwanted reflections.

The first TWT amplifier works at $13.75-14.5 \mathrm{GHz}$ at maximum $700 \mathrm{~W}$.

The second TWT amplifier allows to produce 500-Watts maximum power, in the $8.0-18.0 \mathrm{GHz}$ frequency range.

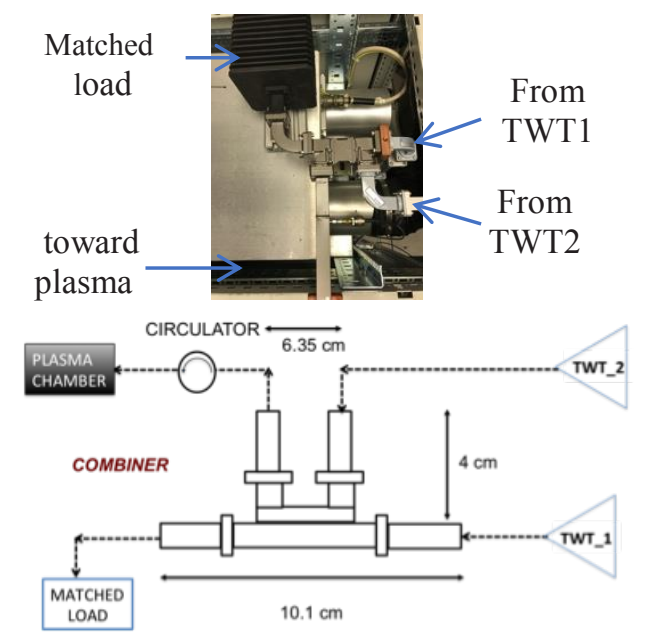

Fig. 1 Microwave scheme for implementing TFH on the CAESAR source at LNS. The same scheme can be used for any ECRIS equipped by only one microwave port for RF matching with the plasma chamber.

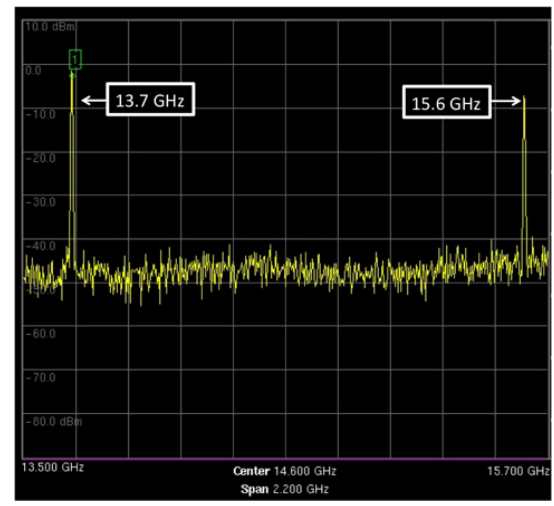

Fig. 2 Spectrum Analyzer Acquisition: $\mathrm{f}_{1}=13.5 \mathrm{GHz} \mathrm{f}_{\text {stop }}=15.7$ $\mathrm{GHz} \mathrm{f}_{\text {center }}=14.6 \mathrm{GHz}$ Span=2.2 GHz.

Before to proceed with the plasma heating, we tested the Experimental setup by using a directional coupler terminated on a waveguide matched load. One port of the directional coupler has been connected to a Spectrum Analyzer Anritsu MS2720T-0743 9 kHz-43 GHz working at Resolution Bandwidth $\mathrm{RBW}=3 \mathrm{MHz}$ and Sweep Time $=200 \mathrm{~ms}$ in Continuous sweep mode.

As it is possible to see in Fig. 2, thanks to the diplexer we are able to simultaneously transmit two frequencies (13.7 $\mathrm{GHz}$ and $15.6 \mathrm{GHz}$ ) without interference and power loss.

\subsection{Comparison of the injector of the Superconducting Cyclotron in double- frequency heating mode with respect to the single frequency mode operation}

The CAESAR source is equipped by two roomtemperature solenoids providing axial confinement, and an hexapole for the radial one. The coils are able to produce a maximum field of $1.2 \mathrm{~T}$ when both are energized by $1100 \mathrm{~A}$ of currents. In order to allow a proper beam extraction, the second solenoid is usually operated at currents not larger than $800 \mathrm{~A}$, so that the mirror configuration is asymmetric. In the specific case of TFH operations, we used Coil 1: 1021 A, Coil 2: 745 A. The extraction potential was fixed at $\mathrm{V}_{\text {extr }}=23726 \mathrm{kV}$; while ${ }^{32} \mathrm{~S}$ charge states were monitored through a magnetic mass spectrometer. The plasma was formed by $\mathrm{SF}_{6}$ gas injected into the plasma chamber at injection pressure of $1.73 \mathrm{E}-6$ mbar. The obtained results are shown in table 1 .

Table 1. ${ }^{32} \mathrm{~S}$ charge states: double-frequency heating mode vs single frequency mode operation.

$\begin{array}{lccc}\text { Charge } & \text { RF power } & \text { Frequency } & \text { Current } \\ \text { state } & {[\mathrm{W}]} & {[\mathrm{GHz}]} & {[\mu \mathrm{A}]}\end{array}$


${ }^{32} \mathrm{~S}^{11+}$

305

14.360

1.0

${ }^{32} \mathrm{~S}^{11+}$

$$
\begin{aligned}
& 380+170 \\
& =550
\end{aligned}
$$

$14.450+$

15.434

Each beam current was optimized at the given frequency (or double frequency) operating mode in order to minimize the reflected power and the beam ripple. Frequency tuning method was adopted for the single frequencies. Results show that when operating in "double frequency" mode, we were able to obtain $\mathrm{I}=2.8$ $\mu \mathrm{A}$ of ${ }^{32} \mathrm{~S}^{11+}$, i.e. a beam current that was almost tripled even though the power was less than doubled. This result is even more relevant if one considers that in ECRIS the beam currents are less than linear dependent on the RF power in single frequency operations.

\section{Innovative launching scheme}

A novel microwave launcher has been electromagnetically characterized to search for the possibly of exciting Electrostatic Bernstein Waves (EBW) inside the prototype installed at INFN-LNS called FPT - Flexible Plasma Trap [11]. In FPT, the plasma can be generated by using two or three different microwave systems, for parallel and perpendicular microwave injections, as figure 3 shows. In particular, we are now working with two TWTs: one operates at a central frequency of $5 \mathrm{GHz}$, in the range 3-7 GHz, and the other around $14 \mathrm{GHz}$ (range $13.75-14.5 \mathrm{GHz}$ ).

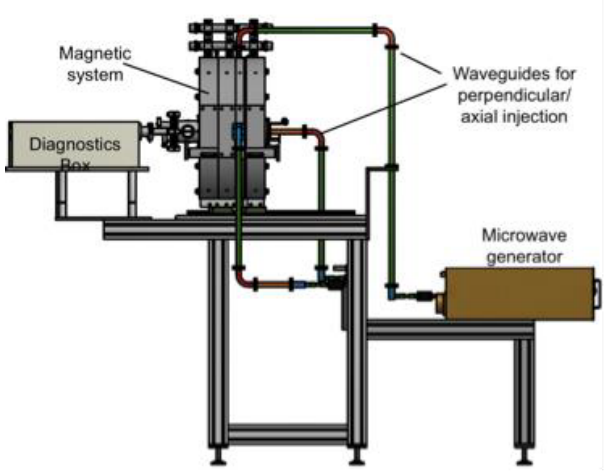

Fig. 3 General layout of the FPT setup.

FPT is equipped by three solenoids providing three different possible profiles of the magnetic field: simple mirror; quasi-flat; and magnetic beach. No beam extraction systems have been coupled to the machine. One of the chamber endplates is dedicated to microwave launching and gas-injection, the other one to plasma diagnostics.

\subsection{Modal-conversion scenario}

The EB waves are electrostatic modes and need to be excited via a inner plasma mode-conversion process, starting from electromagnetic waves (the X mode) at the upper hybrid resonance (UHR) layer, where their generation may involve one (XB) or two mode conversion scenarios $(\mathrm{OXB})$ that are efficient only in a narrow window of the following parameters: frequency, polarization and angle of incidence.

Numerous experiments were performed in fusion plasma toroidal devices where ray tracing approximation allows to compute the electromagnetic waves traveling inside the plasma $[12,13]$. In these "big-size" scenarios, wellestablished optical techniques (e. g. grooved mirrors, lenses and grating polycromator), were adopted to optimize the O-X-B conversion, in terms of wave polarization and incidence angle.

On the contrary, in ECRIS, the pumping wavelength is comparable with the plasma radius [14] and density scale-length, thus prohibiting any ray tracing approach. Full-wave calculations must be applied to simulate the wave behaviour in the plasma $[15,16]$. The presence of resonant cavity additionally causes the polarization scrambling, and it further complicates the OXB modeling. The mechanical constraints also make very difficult the positioning of complex launching systems. The solution hereby proposed consists of a phased waveguide "array" of two elements: using a relative phase shift between them, a steering of the radiated beam can be produced, in order to obtain an optimum incidence angle. The system is described in details in [17].

\subsection{The new microwave launcher based on a two-waveguides-array}

This section is devoted to the microwave transmission line of the heating antenna. Simulations showed O-X-Blike scenarios are possible by properly launching the pumping $\mathrm{O}$-wave from the radial port. As mentioned in the previous section, the antenna should be optimized in order to emit the heating power with an optimal $\mathbf{k}(\mathrm{r})$ angle with respect to the external magnetic field $\mathrm{B} 0$. The absorption of the waves in the vicinity of the resonance layer should be almost complete in a single "pass", if the proper mode of irradiation is chosen.

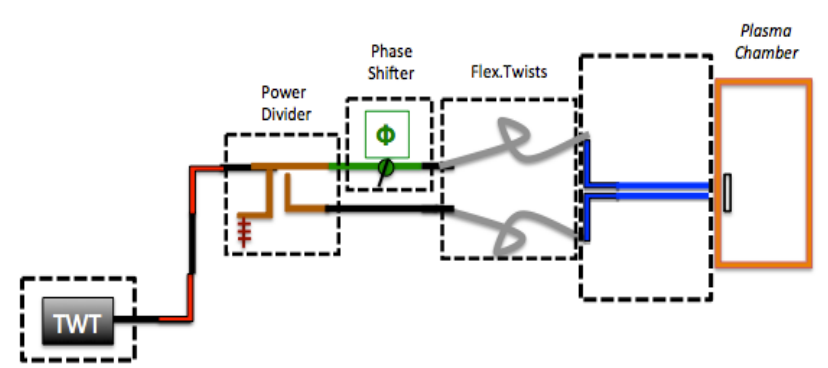

Fig. 4 Microwave transmission line

Taking inspiration from phased multi-waveguide structures (nicknamed waveguide "grill") [17, 18] designed to launch microwaves at the lower hybrid resonance to heat large toroidal plasmas, a doublewaveguide antenna system has been proposed for the 
FPT. The microwave transmission line is schematically shown in fig. 4.

The launcher consists of two waveguide driven in TE10 mode with relative phase controlled by a calibrate phase shifter, a loaded four-port power divider and two fexible and twistable WR62 waveguides.

The $\mathbf{k}$ direction for different phase shift can be simulated, as shown in figure 5. For a $\Delta \phi=0^{\circ}$ (Fig. 5a), the lobe points in forward direction, meaning that the $\mathrm{O}$ wave propagates as purely perpendicular to the magnetic field, while for delta $\Delta \phi=180^{\circ}$ ((Fig. 5b) a two lobes structure is generated, with an angle of $60^{\circ}$ with respect to the magnetostatic field direction.

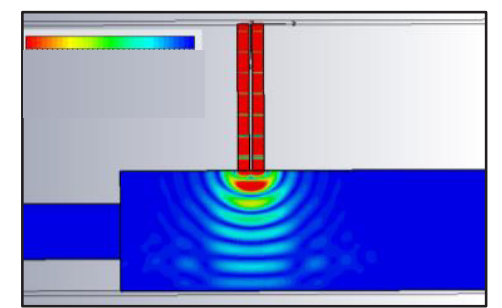

a)

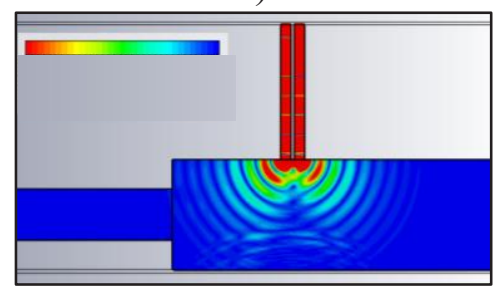

b)

Fig. 5 CST microwave studio "Free-space" vs "In-cavity" simulation of microwave radiated Electric field $|E|$ from the double waveguide array launcher (plots are given with pseudocolor representation of the electric field intensity) a) when the phase shift $=0^{\circ} ; \mathrm{b}$ ) when the phase shift $=180^{\circ}$

\section{Conclusions}

The paper has commented the schemes of microwave-toplasma coupling in ECRIS-type machines. Other than in the framework of ECR-heating of plasmas generated in such machines, efforts are now devoted to new launching schemes, possibly suitable for modalconversion. In the first case, frequency tuning and multiple-frequency heating techniques can be used also in combination, and even in case of very compact and simple machines. For the modal-conversion scenario, difficulties arise due to the critical scale-lengths involved in such compact machines. Nevertheless a phasedwaveguide-array has been designed and tested on a testbench, and it is now going to be implemented for OXB modal-conversion experiments at INFN-LNS.

\section{Acknowledgement}

The authors wish to thank the 5th Nat. Comm. of INFN, under the Grant VESPRI, UTOPIA, RDH, VESPRI, PANDORA for the financial support. M. Castro, S. Marletta, S. Passarello, G. Pastore, S. Vinciguerra, E.
Zappalà, G. Messina. and the INFN-LNS mechanical workshop people are warmly acknowledged for the support given.

\section{References}

1. Geller R., et al. Proc. 8th Int. Workshop ECR Ion Sources, Vol. 1 (1987), p. 22; East Lansing, MIUSA NSCL report MSUCP-47.

2. S. Gammino and G. Ciavola, Plasma Source Sci. Technol., 519 (1996).

3. Z. Q. Xie, Rev. Sci. Instrum. 69, 625 (1998);

4. D. Mascali et al., Rev. Sci. Instrum. 83(2):02A336. (2012)

5. L. Celona, G. Ciavola, F. Consoli, S. Gammino, F. Maimone, D. Mascali, P. Spädtke, K. Tinschert, R. Lang, J. Mäder, J. Roßbach, S. Barbarino, R. S. Catalano, Rev. Sci. Instrum. 79, 2,023305, 2008, 10.1063/1.2841694

6. Z. Q. Xie and C. M. Lyneis, in Proceedings of the 12th International Workshop on ECRIS, Wako, INS-J-182 (Institute for Nuclear Study, University of Tokyo, Japan, 1995), p. 24.

7. V. Skalyga, I. Izotov, T. Kalvas, H. Koivisto, J. Komppula, R. Kronholm, J. Laulainen, D. Mansfeld, and O. Tarvainen, Physics of Plasmas 22, 083509 (2015); doi: 10.1063/1.4928428

8. A. Girard et al., Phys. Rev. E, 62(1), 1182, (2000).

9. G. Castro et al., "Overdense plasma generation in a compact ion source”, Plasma Sources Sci. Technol. 26, 055019, (2017).

10. A Kohn, G Birkenmeier, E Holzhauer, M Ramisch and U Stroth, Plasma Phys. Control. Fusion 52 (2010) 035003 (13pp).

11. S. Gammino et al Journal of Instrumentation, 12, 07, P07027, July 2017.

12. Petrov, Yu. V. and Becoulet and A. Monakhov, , American Physical Society, 41st Annual Meeting of the Division of Plasma Physics, (1999).

13. Van Eester, Plasma Phys. Controlled Fusion. 34:1539-1562, (1992).

14. R Rácz et al, Plasma Sources Sci. Technol. 26 (2017) 075011 (14pp).

15. G. Torrisi, D. Mascali, G. Sorbello, L. Neri, L. Celona, G. Castro, T. Isernia and S. Gammino, J. Electromagnet, Wave. , 28 (9), (2014) pp. 1085 1099.

16. D. Mascali, G. Torrisi, L. Neri, G. Sorbello, G. Castro, L. Celona, and S. Gammino, "Eur. Phys. J. D 69 (2015): 27 DOI: 10.1140/epjd/e2014-50168-5

17. G. Torrisi, G. Sorbello, O. Leonardi, D. Mascali, L. Celona, and S. Gammino, Microw. Opt. Technol. Lett., 58: 2629-2634. doi:10.1002/mop.30117

18. M. Brambilla, NUCLEAR FUSION 16, (1976)

19. S. Bernabei, M A Heald, M Hooke, R W Motley and F J Paoloni and M. Brambilla and W D Getty, NUCLEAR FUSION 175 (1977), 